\title{
FORMULACIÓN MATEMÁTICA DEL DISEÑO DE REDES DE ABASTECIMIENTO.
}

\section{MATHEMATICAL FORMULATION OF WATER SUPPLY NETWORK DESIGN.}

Jesús Rafael Hechavarría Hernández, PhD.

Doctor en Ciencias Técnicas (Cuba).

Docente titular de la Facultad de Arquitectura y Diseño en la Universidad Católica de Santiago de Guayaquil, Ecuador. jesus.hechavarria@cu.ucsg.edu.ec

\section{ARTÍCULO DE INVESTIGACIÓN}

Recibido: 28 de febrero de 2017.

Aceptado: 29 de abril de 2017.

\section{RESUMEN}

La modelación matemática tiene como objetivo fundamental reproducir con la mayor exactitud posible el comportamiento real del sistema físico que representa, es la base que se utiliza en el cálculo hidráulico para simular diferentes estados que se producen en la red de distribución de agua sin tener que llegar a experimentarlos físicamente. El proceso de elaborar un modelo matemático para una red de distribución de agua presupone recopilar toda la información posible acerca de su estructura y funcionamiento y tratarla posteriormente para que pueda ser asimilada por los programas de análisis correspondientes. El modelo matemático permite calcular todas las variables que caracterizan su comportamiento, al tener en cuenta el conjunto de líneas y nodos que representan a las tuberías, las bombas y estaciones de bombeo, las válvulas reguladoras y las automáticas, los depósitos y embalses, y los puntos de consumo o inyección que forma la red. La formulación matemática es aplicada a redes ramificadas, malladas y mixtas, al emplear el Método del Gradiente para realizar el cálculo del modelo hidráulico. El caso de estudio presentado muestra la diversidad de criterios a tener en cuenta durante el diseño de una red de abastecimiento, los cuales deben considerarse en el modelo matemático.

Palabras clave: modelación hidráulica, diseño de redes de abasto. 


\section{ABSTRACT}

The main purpose of mathematical modeling is to reproduce the actual behavior of the physical system it represents, it is the basis used in the hydraulic calculation to simulate different states that occur in the water distribution network without having to experience them physically. The process of elaborating a mathematical model for a water distribution network presupposes gathering all possible information about its structure and functioning and treating it later so that it can be assimilated by the corresponding analysis programs. The mathematical model allows to calculate all the variables that characterize its behavior, taking into account the set of lines and nodes that represent the pipes, the pumping stations, the valves, the reservoirs and the points of consumption or injection forming the network. The mathematical formulation is applied to branched, meshed and mixed networks, using the Gradient Method to calculate the hydraulic model. The case study presented shows the diversity of criteria to be taken into account during the design of a supply network, which should be considered in the mathematical model.

Keywords: hydraulic modeling, water supply networks design.

\section{INTRODUCCIÓN}

Un sistema de distribución de agua es un conjunto de elementos encargados de transportar el fluido líquido desde los puntos de suministro hasta los puntos de consumo. Está constituido por tuberías y otros elementos como son: válvulas, elementos de unión, elementos de medición, entre otros, que deben ser dimensionados adecuadamente para suministrar los caudales demandados, para lo cual será necesario mantener en la red unas presiones adecuadas (Aguirre, 1996). El resto de los componentes del sistema de distribución (depósitos, estaciones de bombeo, forma de regulación, entre otros) condicionarán su diseño y cálculo, por lo que en general no se puede tratar la red como un elemento aislado.

Para el dimensionamiento hidráulico de la red se utiliza habitualmente modelos de régimen permanente y se busca que el sistema trabaje incluso en las condiciones más críticas de servicio, por lo que se realiza la simulación hidráulica con el valor del caudal en el horario de mayor demanda (QMH) (Hechavarría et al., 2006). Dada la complejidad y variedad de circunstancias que pueden presentarse en la concepción de una red de distribución de agua, es difícil concebir una metodología general que satisfaga todas las exigencias de diseño (Walski, 2001). 
Para la resolución de sistemas de distribución de agua es necesario utilizar, además de las ecuaciones básicas, otras ecuaciones que permitan relacionar las pérdidas de carga continuas con las características del flujo y el caudal circulante, así como ecuaciones para determinar las pérdidas localizadas.

\section{REVISIÓN TEÓRICA}

\section{Ecuación fundamental de pérdidas de carga.}

Para un movimiento permanente y uniforme de un fluido incompresible, la pérdida de carga unitaria j [metros columna de agua (m.c.a.) / metros de tubería (m)] está relacionada con parámetros geométricos, cinemáticos y las propiedades del fluido. La ecuación fundamental para las pérdidas de carga puede determinarse por Análisis Dimensional al relacionar todos los parámetros (Hechavarría, 2009; Montero, 2016). La ecuación de Darcy-Weisbach expresa:

$$
j=\frac{f}{D} \frac{v^{2}}{2 g}
$$

El parámetro $f$ se denomina factor de fricción y es función de dos parámetros adimensionales: el número de Reynolds $(\mathrm{Re})$, que expresa la relación entre las fuerzas de inercia y las fuerzas viscosas y la rugosidad equivalente la cual está en función del diámetro y la rugosidad del material de la tubería.

Debido a los orígenes no empíricos, la ecuación de Darcy-Weisbach es considerada por muchos ingenieros como el método más preciso para modelar pérdidas de fricción. Se describe comúnmente de la manera siguiente (Streeter et al., 2000):

$$
h_{f}=f \frac{L}{D} \frac{v^{2}}{2 g}
$$

Donde:

hf: pérdida de carga del tramo $(\mathrm{m})$.

f: factor de fricción de Darcy-Weisbach (adimensional).

D: diámetro de la tubería (m).

$\mathrm{L}$ : longitud de la tubería $(\mathrm{m})$.

v: velocidad del flujo $(\mathrm{m} / \mathrm{s})$.

$\mathrm{g}$ : aceleración de la gravedad (m/s2). 
Colebrook \& White combinaron las expresiones de Von Karman-Prandtl para conductos lisos y rugosos y obtuvieron una buena aproximación para el régimen turbulento de transición en tuberías comerciales, lo cual permite calcular el valor del factor de fricción de DarcyWeisbach para cualquier régimen turbulento (Hechavarría 2007). La ecuación de Colebrook \& White expresa:

Donde:

$$
\frac{1}{\sqrt{f}}=-2 \log \left(\frac{\varepsilon}{3,7 D}+\frac{2,51}{\operatorname{Re} \sqrt{f}}\right)
$$

f: factor de fricción de Darcy-Weisbach (adimensional).

$\mathrm{E}$ : coeficiente de rugosidad de la tubería $(\mathrm{m})$.

D: diámetro de la tubería $(m)$.

El factor de fricción está en función del número de Reynolds del flujo, el cual depende de la velocidad del fluido y ésta a su vez de la descarga. La ecuación de Colebrook \& White, implícita para f, se resuelve iterativamente por ensayo y error, con la aplicación de algún método de aproximaciones sucesivas (Hechavarrìa, 2010). Como primera aproximación para determinar el factor de fricción de Darcy-Weisbach puede utilizarse la ecuación de Swamee \& Jain (Haestad, 2009).

$$
f=\frac{1,325}{\left[\ln \left(\frac{\varepsilon}{3,7 D}+\frac{5,74}{\mathrm{Re}^{0,9}}\right)\right]^{2}}
$$

Las pérdidas de energía locales pueden surgir en áreas localizadas de turbulencia por variaciones en la sección transversal de las tuberías por la presencia de elementos como: válvulas, cambios bruscos de dirección, entre otros. En ocasiones se consideran despreciables, en comparación a las pérdidas por fricción, por lo que serían descartadas del análisis. Cuando en un sistema de tuberías las pérdidas locales son numerosas, con relación a la longitud de éstas, las caídas singulares de energía tendrían un impacto significativo en las pérdidas totales del sistema. En ese caso se expresan como una fracción de altura de velocidad a diámetro nominal (caso válvulas y codos) aunque en secciones de diámetro variable (estrechamientos y ensanchamientos) casi siempre se especifica el diámetro a que está referida dicha fracción (Hechavarría, 2009). 
Donde:

$$
h_{l}=k \frac{v^{2}}{2 g}
$$

hl: pérdida de energía local $(\mathrm{m})$

$\mathrm{k}$ : coeficiente adimensional en función del Re y los parámetros del elemento.

v: velocidad del flujo $(\mathrm{m} / \mathrm{s})$.

g: aceleración de la gravedad (m/s2).

En el diseño de una red de distribución de agua intervienen variables de naturaleza hidráulica, que caracterizan funcionalmente el sistema, y variables de tipo económico que determinan los costos asociados a cada uno de los elementos de la red, así como su relación con las variables hidráulicas.

\section{Relaciones y restricciones de naturaleza hidráulica.}

Desde el punto de vista topológico, una red de distribución de agua se compone de nodos y líneas. Los nodos corresponden a los puntos del sistema donde físicamente se produce una aportación o un consumo de caudal, o pueden ser simplemente puntos de conexión donde confluyen varias tuberías. Los nodos están unidos entre sí mediante líneas, que identifican elementos de transporte (tuberías) o cualquier elemento singular que provoque cambios en la energía específica del fluido (por ejemplo: bombas y/o válvulas) (Aguirre et al., 1996; Hechavarría et al. 2010).

Cada línea queda caracterizada hidráulicamente por una serie de variables; en el caso de una línea que corresponde a una tubería, las cuatro variables hidráulicas fundamentales son:

velocidad $\mathrm{v}$, caudal $\mathrm{Q}$, diámetro $\mathrm{D}$, y pérdida de carga hf. Existen dos ecuaciones básicas que ligan obligatoriamente estos cuatro parámetros en cada línea, la ecuación de continuidad y la ecuación de pérdidas (Hechavarría, 2011):

Ecuación de continuidad:

$$
Q=\frac{\pi D^{2}}{4} v
$$

Donde:

Q: caudal del líquido (I/s).

D: diámetro de la tubería (m). 
v: velocidad del flujo $(\mathrm{m} / \mathrm{s})$.

La ecuación de continuidad se plantea como la relación $Q=f(v, D)$ para el caso de conductos de sección circular. En el caso de la ecuación de pérdidas se recomienda utilizar la ecuación de Darcy-Weisbach porque es consistente dimensionalmente y es la más utilizada con este fin.

En un modelo de análisis en régimen permanente de una red hidráulica mallada se hace uso de dos leyes generales, que se cumplen independientemente de la configuración y los elementos que componen la red y que constituyen una particularización de las ecuaciones generales de conservación de la masa y la energía aplicada al flujo de un fluido incompresible a través de un sistema de tuberías a presión.

La característica común a todas las formulaciones expuestas es que el sistema de ecuaciones a resolver es no lineal, al menos en una parte de las ecuaciones, característica que viene determinada no por la propia formulación de las leyes de equilibrio, sino por el comportamiento no lineal que siguen los elementos que componen el sistema. No existe hasta el presente ningún método de resolución directa y es por tanto necesario recurrir a métodos iterativos. El Método del Gradiente resuelve simultáneamente las ecuaciones de continuidad en los nodos y las ecuaciones de comportamiento hidráulico de las tuberías a presión para un instante dado.

\section{Método del Gradiente.}

El Método del Gradiente es un eficiente método de cálculo hidráulico que implementa un modelo para la resolución de sistemas de tuberías a presión, representado por un sistema de ecuaciones lineales expresadas en forma matricial, tiene como principal ventaja que evita el ensamblado de las matrices por lo que disminuye la cantidad de procesos a realizar en comparación con otros métodos. Aspecto muy importante durante los procedimientos de optimización.

A continuación, se enuncian algunas de las ventajas de la implementación computacional del Método del Gradiente aplicado a los sistemas de tuberías a presión:

1. Asegura solución única al no tener problemas de convergencia, debido a que la solución del sistema de ecuaciones a resolver, pasa por obtener la inversa de una matriz simétrica y definida positiva. 
2. Permite expresar la topología de la red, las pérdidas de carga y la continuidad de caudales, en términos de ecuaciones matriciales.

3. Emplea un modelo real de redes, al incorporar el concepto de las matrices de conectividad.

4. No es necesario estimar una solución inicial cercana al valor real.

5. Se ingresa una cantidad mínima de datos relativos a los nodos y tramos de la red, y no ecuaciones explícitas.

6. Puede ser aplicado a redes malladas $y / 0$ ramificadas. Lo cual se considera ventajoso comparado con otros algoritmos iterativos, que requieren una reformulación de la red a un conjunto de circuitos equivalentes.

7. Permite implementar matrices huecas en la solución de sistemas de ecuaciones simultáneas, las que hacen el cálculo extremadamente rápido y seguro.

\section{Descripción del Método del Gradiente.}

Sea una red de tuberías con N nodos y NF nodos de altura dada (embalses y depósitos). La relación entre la pérdida de carga para una tubería que va del nodo i al nodo j, y el caudal de paso Qij, puede escribirse como (Rossman, 2001):

$$
H_{i}-H_{j}=h_{i j}=r Q_{i j}^{n}+m Q_{i j}^{2}
$$

Donde:

Hi: altura piezométrica en el nodo.

$h$ : pérdida de carga.

r. coeficiente de resistencia.

Q: caudal.

$n$ : exponente del caudal.

$m$ : coeficiente de pérdidas menores.

El valor del coeficiente de resistencia depende de la fórmula utilizada para el cálculo de las pérdidas. Para las bombas, la pérdida puede representarse mediante una fórmula potencial del tipo:

$$
h_{i j}=-\omega^{2}\left(h_{0}-r\left(\frac{Q_{i j}}{\omega}\right)^{n}\right)
$$

Donde:

ho: altura a caudal nulo.

W: velocidad relativa de giro. 
r. coeficiente de la curva de la bomba.

$n$ : coeficiente de la curva de la bomba.

Los términos reconocidos como, coeficiente de la curva de la bomba, $r$ y $n$, son referenciados por otros autores como: coeficiente de resistencia y exponente del caudal respectivamente. En la práctica, al proporcionar la altura a válvula cerrada ho y otros dos puntos de la curva característica de una bomba, se pueden calcular estos valores.

El segundo sistema de ecuaciones a cumplir está configurado por la condición de equilibrio para los caudales en todos los nodos:

$$
\sum_{i=1}^{n} Q_{i j}-D_{i}=0
$$

Donde:

Di:caudal de demanda en el nodo $i$.

El cual por convención se toma como positivo cuando entra al nodo. Dados los valores de las alturas en los nodos de altura prefijada, se trata de encontrar una solución para las alturas $\mathrm{Hi}$ en los restantes nodos, y para los caudales Qij de todos los tramos de tuberías, que satisfagan las ecuaciones (Hechavarría, 2009; Montero et al. 2016).

El método de resolución del Gradiente comienza con una estimación inicial del caudal por cada tubería, sin necesidad de cumplir la ecuación de continuidad. En cada iteración del método, se obtienen las alturas piezométricas en los nodos y se resuelve el sistema de ecuaciones:

$$
A H=F
$$

Donde:

A: matriz Jacobiana $(N \times N)$.

$H$ : vector de incógnitas nodales $(N \times 1)$.

$F$ : vector de términos independientes $(N \times 1)$.

Los elementos de la diagonal principal de la matriz Jacobiana vienen dados por:

$$
A_{i i}=\sum_{j} p_{i j}
$$

y los elementos no nulos fuera de la diagonal principal, por: 


$$
A_{i j}=-p_{i j}
$$

Donde:

pij: la inversa de la derivada respecto al caudal, de la pérdida de carga en la línea que va del nodo i al j. Su expresión para las tuberías es:

$$
p_{i j}=\frac{1}{n r\left|Q_{i j}\right|^{n-1}+2 m\left|Q_{i j}\right|}
$$

y para las bombas:

$$
p_{i j}=\frac{1}{n \omega^{2} r\left(\frac{Q_{i j}}{\omega}\right)^{n-1}}
$$

Los términos independientes están constituidos por el caudal residual en el nodo no equilibrado, más un factor de corrección dado por:

$$
F_{i}=\left(\sum_{j} Q_{i j}-D_{i}\right)+\sum_{j} y_{i j}+\sum_{f} p_{i f} H_{f}
$$

Donde:

pifHf: sólo para tuberías que conectan el nodo i con un nodo de altura conocida $f$. yiff: factor de corrección del caudal yif.

La expresión del factor de corrección del caudal yif para las tuberías donde:

$$
\operatorname{sgn}(x)=\left\{\begin{array}{r}
1, \quad \text { Si } x>0 \\
-1, \quad \text { en otro caso }
\end{array}\right.
$$

se define como:

$$
y_{i j}=p_{i j}\left(r\left|Q_{i j}\right|^{n}+m\left|Q_{i j}\right|^{2}\right) \operatorname{sgn}\left(Q_{i j}\right)
$$

para las bombas

$$
y_{i j}=-p_{i j} \omega^{2}\left(h_{0}-r\left(\frac{Q_{i j}}{\omega}\right)^{n}\right)
$$

Donde:

$Q_{i j}$ : siempre es positivo.

Una vez calculadas las nuevas alturas, se resuelven las ecuaciones (10), los nuevos caudales se obtienen mediante la expresión: 


$$
Q_{i j}=Q_{i j}-\left(y_{i j}-p_{i j}\left(H_{i}-H_{j}\right)\right)
$$

Si la suma, extendida a todas las líneas, del valor absoluto de la variación relativa del caudal respecto al caudal total en la línea, es mayor que una cierta tolerancia (p. ej. 0,001), las ecuaciones (10) y (18) se resuelven de nuevo. Obsérvese que la fórmula de actualización (18) conduce al equilibrio de los caudales en los nodos, tras la primera iteración. La descripción del método, así como la implementación pueden ser consultadas en (Rossman, 2001).

\section{ANÁLISIS DE RESULTADOS}

\section{Análisis de un caso de estudio de una red de abastecimiento.}

En la figura 1 se muestra el trazado concebido para el diseño de la red de distribución de agua. En la figura 2 y las tablas 1 y 2, se ilustran los resultados del cálculo hidráulico. 
Figura 1. Trayectoria de la red de abastecimiento de agua potable.

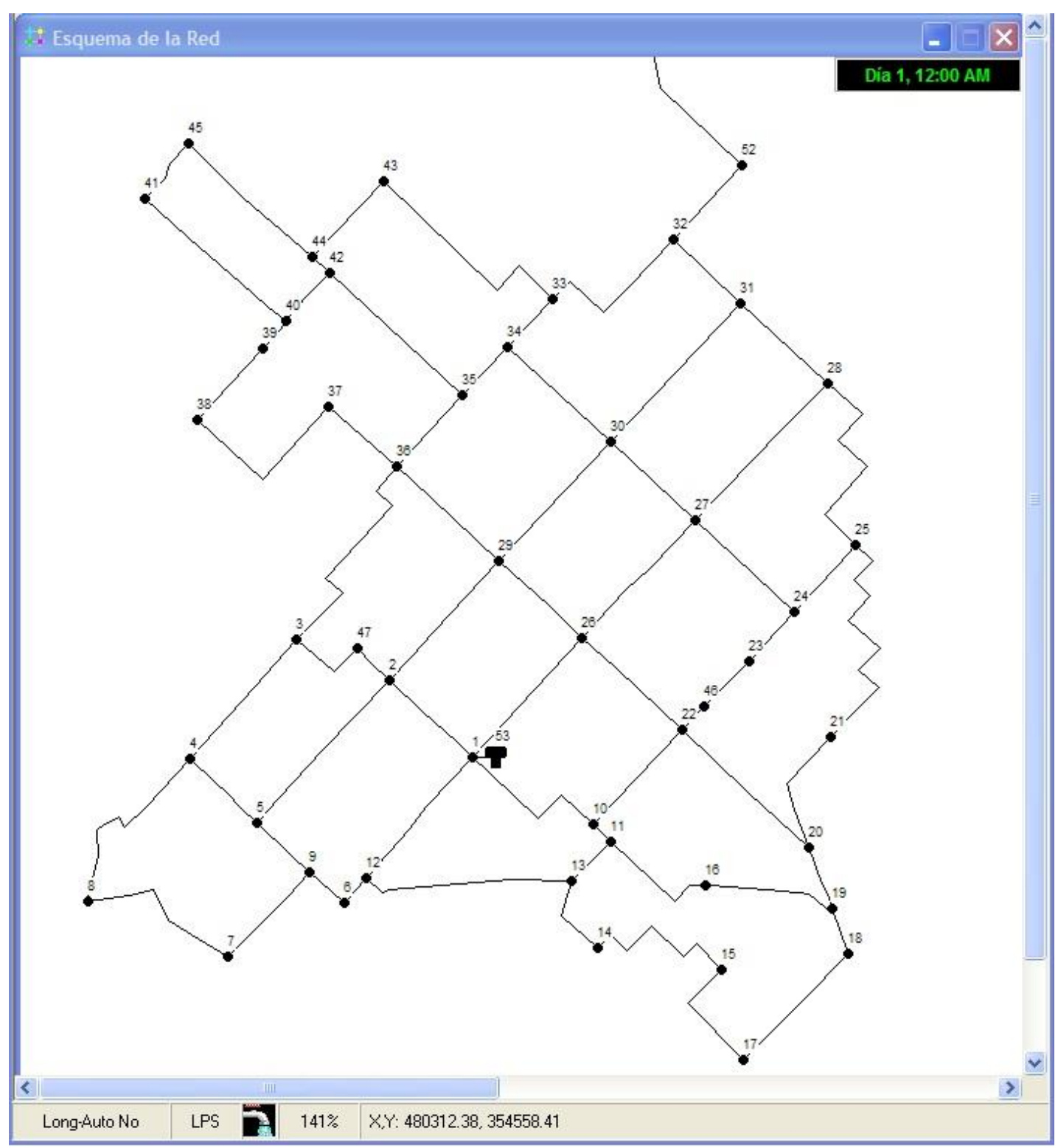

Fuente: elaboración propia. 
Figura 2. Simulación hidráulica en EPANET de la red de distribución de agua potable.

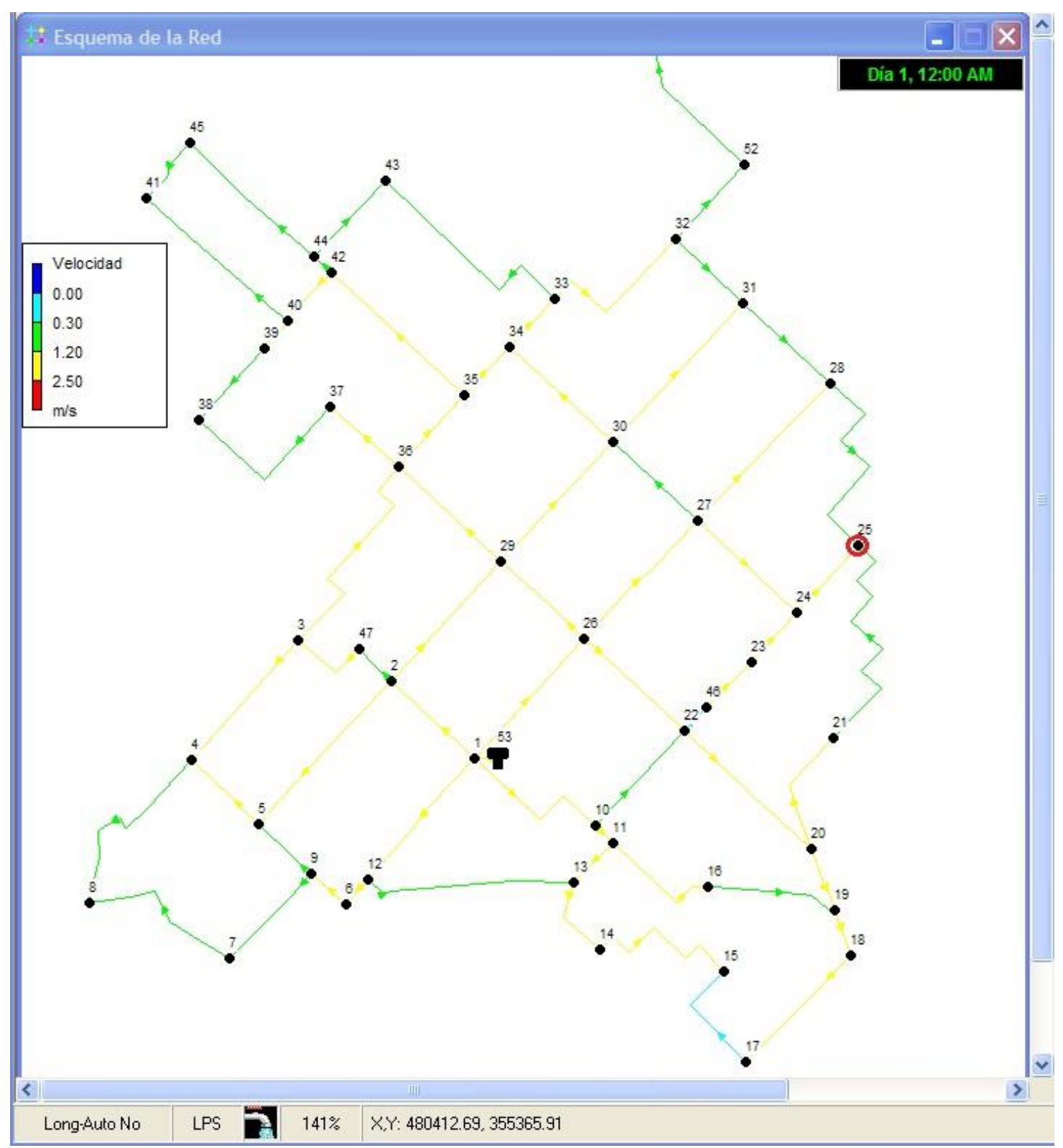

Fuente: elaboración propia. 
Tabla 1: Resultados obtenidos en los tramos de tubería con EPANET.

\begin{tabular}{|c|c|c|c|c|c|c|}
\hline & Longitud & Diámetro & Rugosidad & Caudal & Velocidad & Pérdida Unitaria \\
\hline ID Línea & $<m>$ & $<\mathrm{mm}>$ & & $\langle 1 / s\rangle$ & $\langle\mathrm{m} / \mathrm{s}\rangle$ & $<\mathrm{m} / \mathrm{km}>$ \\
\hline Tubería 1 & 333,56 & 290,8 & 145 & 102 & 1,54 & 6,34 \\
\hline Tubería 2 & 333,34 & 147,6 & 145 & 6,67 & 0,39 & 1,1 \\
\hline Tubería 3 & 637,84 & 101,6 & 145 & 8,98 & 1,11 & 11,81 \\
\hline Tubería 4 & 313,02 & 147,6 & 145 & 24,63 & 1,44 & 12,41 \\
\hline Tubería 5 & 5969,0 & 831,2 & 145 & 1040,22 & 1,92 & 2,81 \\
\hline Tubería 6 & 646,90 & 83,0 & 145 & 0,41 & 0,08 & 0,1 \\
\hline Tubería 7 & 688,04 & 83,0 & 145 & 0,82 & 0,15 & 0,38 \\
\hline Tubería 8 & 533,17 & 83,0 & 145 & 1,23 & 0,23 & 0,8 \\
\hline Tubería 9 & 595,53 & 83,0 & 145 & 1,64 & 0,3 & 1,36 \\
\hline Tubería 10 & 347,61 & 147,6 & 145 & 16,56 & 0,97 & 5,95 \\
\hline Tubería 11 & 284,04 & 369,4 & 145 & 169,31 & 1,58 & 5,05 \\
\hline Tubería 12 & 153,24 & 500,0 & 145 & 169,31 & 0,86 & 1,16 \\
\hline Tubería 13 & 219,31 & 101,6 & 145 & 14,57 & 1,8 & 28,94 \\
\hline Tubería 14 & 112,03 & 300,0 & 130 & 14,57 & 0,21 & 0,18 \\
\hline Tubería 15 & 824,46 & 290,8 & 145 & 143,4 & 2,16 & 11,91 \\
\hline Tubería 16 & 560,54 & 461,8 & 145 & 402,27 & 2,4 & 8,46 \\
\hline Tubería 17 & 469,74 & 327,8 & 145 & 139,43 & 1,65 & 6,31 \\
\hline Tubería 18 & 359,05 & 101,6 & 145 & 3,61 & 0,45 & 2,19 \\
\hline Tubería 19 & 816,63 & 101,6 & 145 & 5,13 & 0,63 & 4,18 \\
\hline Tubería 20 & 227,06 & 184,6 & 145 & 39,61 & 1,48 & 10,06 \\
\hline Tubería 21 & 791,42 & 101,6 & 145 & 4,12 & 0,51 & 2,79 \\
\hline Tubería 22 & 407,60 & 147,6 & 145 & 9,74 & 0,57 & 2,22 \\
\hline Tubería 23 & 654,63 & 147,6 & 145 & 36,64 & 2,14 & 25,89 \\
\hline Tubería 24 & 564,74 & 290,8 & 145 & 150,69 & 2,27 & 13,06 \\
\hline Tubería 25 & 467,07 & 184,6 & 145 & 55,1 & 2,06 & 18,53 \\
\hline
\end{tabular}


Revista Científica ECOCIENCIA

\begin{tabular}{|c|c|c|c|c|c|c|}
\hline Tubería 26 & 396,90 & 147,6 & 145 & 13,39 & 0,78 & 4,01 \\
\hline Tubería 27 & 589,35 & 166,2 & 145 & 34,48 & 1,59 & 12,97 \\
\hline Tubería 28 & 316,34 & 101,6 & 145 & 3,0 & 0,37 & 1,55 \\
\hline Tubería 29 & 651,91 & 166,2 & 145 & 46,04 & 2,12 & 22,16 \\
\hline Tubería 30 & 482,68 & 230,8 & 145 & 64,43 & 1,54 & 8,34 \\
\hline Tubería 31 & 564,05 & 290,8 & 145 & 137,37 & 2,07 & 11 \\
\hline Tubería 32 & 559,54 & 327,8 & 145 & 169,76 & 2,01 & 9,08 \\
\hline Tubería 33 & 390,46 & 230,8 & 145 & 58,94 & 1,41 & 7,07 \\
\hline Tubería 34 & 478,52 & 184,6 & 145 & 46,0 & 1,72 & 13,27 \\
\hline Tubería 35 & 229,53 & 184,6 & 145 & 34,31 & 1,28 & 7,71 \\
\hline Tubería 36 & 124,74 & 147,6 & 145 & 22,32 & 1,3 & 10,33 \\
\hline Tubería 37 & 622,64 & 230,8 & 145 & 67,69 & 1,62 & 9,14 \\
\hline Tubería 38 & 222,82 & 184,6 & 145 & 36,82 & 1,38 & 8,78 \\
\hline Tubería 39 & 81,58 & 101,6 & 145 & 9,11 & 1,12 & 12,12 \\
\hline Tubería 40 & 579,2 & 101,6 & 145 & 5,5 & 0,68 & 4,76 \\
\hline Tubería 41 & 643,64 & 147,6 & 145 & 14,5 & 0,85 & 4,65 \\
\hline Tubería 42 & 253,75 & 101,6 & 145 & 5,5 & 0,68 & 4,76 \\
\hline Tubería 43 & 231,48 & 101,6 & 145 & 14,57 & 1,8 & 28,94 \\
\hline Tubería 44 & 314,22 & 101,6 & 145 & 15,75 & 1,94 & 33,42 \\
\hline Tubería 45 & 933,95 & 101,6 & 145 & 4,6 & 0,57 & 3,41 \\
\hline Tubería 46 & 222,7 & 230,8 & 145 & 87,15 & 2,08 & 14,6 \\
\hline Tubería 47 & 597,74 & 290,8 & 145 & 117,57 & 1,77 & 8,25 \\
\hline Tubería 48 & 447,5 & 300 & 130 & 52,83 & 0,75 & 1,97 \\
\hline Tubería 49 & 457,78 & 101,6 & 145 & 15,84 & 1,95 & 33,75 \\
\hline Tubería 50 & 434,39 & 184,6 & 145 & 7,95 & 0,3 & 0,51 \\
\hline Tubería 51 & 511,77 & 184,6 & 145 & 39,47 & 1,47 & 9,99 \\
\hline Tubería 52 & 453,69 & 147,6 & 145 & 15,36 & 0,9 & 5,17 \\
\hline Tubería 53 & 169,21 & 230,8 & 145 & 70,99 & 1,7 & 9,98 \\
\hline Tubería 54 & 604,8 & 147,6 & 145 & 23,57 & 1,38 & 11,43 \\
\hline
\end{tabular}

ISSN: 1390-9320, Vol. 4, No. 3, junio 2017 
Revista Científica ECOCIENCIA

\begin{tabular}{|c|c|c|c|c|c|c|}
\hline Tubería 55 & 436,4 & 166,2 & 145 & 35,54 & 1,64 & 13,72 \\
\hline Tubería 56 & 288,01 & 147,6 & 145 & 29 & 1,69 & 16,79 \\
\hline Tubería 57 & 574,98 & 300 & 130 & 139,25 & 1,97 & 11,86 \\
\hline Tubería 58 & 81,83 & 230,8 & 145 & 66,57 & 1,59 & 8,86 \\
\hline Tubería 59 & 731,79 & 101,6 & 145 & 5,94 & 0,73 & 5,49 \\
\hline Tubería 60 & 189,76 & 147,6 & 145 & 31,03 & 1,81 & 19,03 \\
\hline Tubería 61 & 554,68 & 184,6 & 145 & 45,25 & 1,69 & 12,87 \\
\hline Tubería 62 & 111,46 & 166,2 & 145 & 36,77 & 1,69 & 14,61 \\
\hline Tubería 63 & 247,93 & 101,6 & 145 & 5,66 & 0,7 & 5,02 \\
\hline Tubería 64 & 407,64 & 147,6 & 145 & 17,33 & 1,01 & 6,47 \\
\hline Tubería 65 & 158,51 & 166,2 & 145 & 36,77 & 1,69 & 14,61 \\
\hline Tubería 66 & 587,27 & 101,6 & 145 & 7,82 & 0,96 & 9,13 \\
\hline Tubería 67 & 553,21 & 101,6 & 145 & 12,92 & 1,59 & 23,14 \\
\hline Tubería 68 & 706,35 & 147,6 & 145 & 11,82 & 0,69 & 3,19 \\
\hline Tubería 69 & 321,08 & 101,6 & 145 & 9,86 & 1,22 & 14,03 \\
\hline Tubería 70 & 671,26 & 166,2 & 145 & 36,97 & 1,7 & 14,76 \\
\hline Tubería 71 & 391,77 & 500 & 145 & 418,34 & 2,13 & 6,17 \\
\hline \multicolumn{6}{|l|}{ Total } & 696,86 \\
\hline
\end{tabular}

Fuente: elaboración propia. 
Tabla 2: Resultados en los Nodos (EPANET).

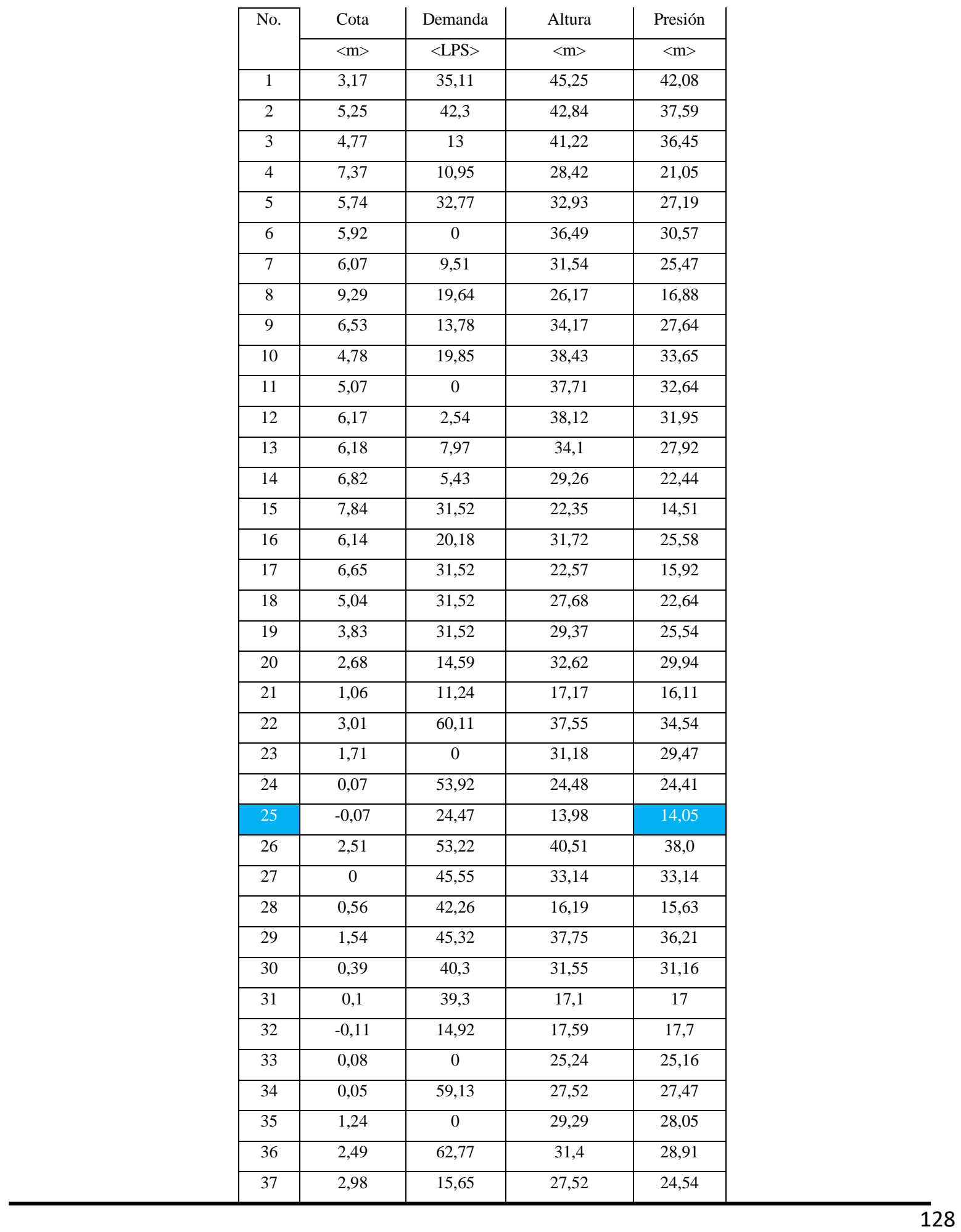

ISSN: 1390-9320, Vol. 4, No. 3, junio 2017 
Fuente: elaboración propia.

\begin{tabular}{|c|c|c|c|c|}
38 & 5,28 & 15,65 & 19,98 & 14,7 \\
\hline 39 & 4,82 & 15,65 & 20,35 & 15,53 \\
\hline 40 & 3,15 & 0 & 21,64 & 18,49 \\
\hline 41 & 4,48 & 20 & 18,65 & 14,17 \\
\hline 42 & 2,37 & 21,76 & 23,6 & 21,23 \\
\hline 43 & 0,61 & 8,74 & 21,82 & 21,21 \\
\hline 44 & 2,14 & 0 & 22,61 & 20,47 \\
\hline 45 & 3,14 & 0 & 19,85 & 16,71 \\
\hline 46 & 2,64 & 0 & 37,53 & 34,89 \\
\hline 47 & 6,15 & 0 & 42,66 & 36,51 \\
\hline 48 & 0,1 & 0,41 & 13,97 & 13,87 \\
\hline 49 & 0,27 & 0,41 & 14,03 & 13,76 \\
\hline 50 & $-0,47$ & 0,41 & 14,29 & 14,76 \\
\hline 51 & $-0,53$ & 0,41 & 14,72 & 15,25 \\
\hline 52 & 0,08 & 14,92 & 15,52 & 15,44 \\
\hline
\end{tabular}

\section{Requerimientos establecidos para la simulación hidráulica.}

Al considerar los valores mínimos, intermedios y máximos de velocidad y presión requeridos por los diseñadores, se realiza la simulación del cálculo hidráulico a partir de una escala de colores. Para los resultados inferiores al valor mínimo, se establece el color (azul claro), para los superiores al máximo color (rojo) y para los resultados admisibles color (verde) y (amarillo), de menor a mayor valor. En la tabla 3 se muestran los valores admisibles, escogidos para la modelación.

Tabla 3: Requerimiento de velocidad y presión.

Fuente: elaboración propia.

\begin{tabular}{|l|c|c|c|}
\hline Requerimiento & Mínimo & Intermedio & Máximo \\
\hline Velocidad (m/s) & 0,3 & 1,2 & 2,5 \\
\hline Presión (mca) & 14,0 & 20,0 & 50,0 \\
\hline
\end{tabular}

En la figura 2 se destaca la ubicación del punto crítico con un círculo de color rojo que indica el punto de menor valor de presión en el sistema hidráulico, nodo 25. La identificación del punto crítico en sistemas de abastecimiento es de gran importancia para 
poder simular, en caso de incendios, el incremento de la demanda en el nodo. El sistema debe garantizar un determinado valor de presión en el punto crítico bajo esas condiciones.

\section{Topología de la red de abasto del caso de estudio.}

La red de distribución de agua potable caso de estudio se considera topológicamente una red mixta por presentar circuitos y ramificaciones. Lo cual no es un inconveniente para el Sistema CAD, al emplear como método de cálculo hidráulico el Método del Gradiente; el cual, no requiere reformular la red a un conjunto de circuitos equivalentes como hacen otros métodos iterativos aplicados al cálculo de redes mixtas.

La representación topológica que realiza el método en términos de ecuaciones matriciales, así como, la implementación de matrices huecas en la solución del sistema de ecuaciones simultáneas, hacen que el proceso de cálculo sea rápido y seguro. Aspecto importante fundamentalmente en procesos de optimización con gran cantidad de iteraciones.

\section{Inserción de la tarea de estudio al Sistema de Mayor Envergadura.}

Al insertar la tarea en estudio al Sistema de Mayor Envergadura se consideran que el ramal con longitud de 2463,64 m a partir del nodo 52 y que se describe sucesivamente con los nodos 51, 50, 49 y 48, no será considerado de mayor importancia que la red mallada, que abarca la totalidad de la ciudad. Este planteamiento se basa, en que el ramal descrito transportará una pequeña porción del caudal demandado por la red. Sin embargo, será necesario garantizar presiones "aceptables" durante el cálculo hidráulico. Para enriquecer la ilustración de los resultados del cálculo hidráulico, se destacan los nodos y tramos referentes al ramal, con un color verde, ver tablas 1 y 2 , además de la tabla 3. Los demás colores utilizados para destacar los valores de velocidad y presión se corresponden con los establecidos para la simulación hidráulica.

Un análisis preliminar a este ramal, trajo como resultado que el diámetro interior mínimo de las tuberías debe ser de $83,0 \mathrm{~mm}$, Diámetro Nominal (DN) 90, precisamente por el análisis del caudal a incrementar en caso de incendio. En este caso se toma la decisión de garantizar valores de presión "aceptables" en los nodos del ramal, a pesar de obtener

ISSN: 1390-9320, Vol. 4, No. 3, junio 2017 
como resultado del cálculo hidráulico valores de velocidad inferiores a los admisibles para las condiciones de máxima demanda horaria, $\mathrm{QMH}$.

También se tuvo en cuenta 545,01 m de tuberías de Fibra de Vidrio (FV), de DN 500, descrito por los nodos 1, 2 y 47 (dos tramos de tuberías) y 1134,52 m de tuberías de Hierro Fundido (HoFo), de DN 300, descrito por los nodos 1, 10, 22 y 46 (tres tramos de tuberías), el resto de las tuberías corresponden al material PEAD.

En el caso de estudio no se considera el diseño del tanque elevado pero un análisis preliminar de la conducción arrojó como resultado que se garantizará una presión de 42,08 m en el nodo 1 (Suministro), al cual se transportará 1040,22 L/s que demanda la red, desde una distancia de 5969,0 m por una tubería de PEAD de DN 900, diámetro interior de $831,2 \mathrm{~mm}$. Las pérdidas unitarias en la conducción se computan en 2,81 m/km, ver tabla 1 (tubería 5), lo que representa unas pérdidas energéticas por fricción evaluadas en $16,77 \mathrm{~m}$.

\section{DISCUSIÓN}

\section{Aspectos relevantes de la solución de diseño obtenida.}

La solución de diseño de la red de distribución de agua potable tiene como resultados relevantes que el menor valor de presión computado en la red mallada, nodo 25 "punto crítico", es superior al permisible, al considerar los valores de presión obtenidos en los nodos 48 y 49 "aceptables". Por otra parte, el menor valor de velocidad, tubería 58, tramo 22-46, a pesar de ser inferior al mínimo permisible, se considera “aceptable”. La presencia de velocidades inferiores a las permisibles trae consigo analizar medidas prácticas como es la proyección de válvulas de purga para drenar la red cada cierto período de tiempo, debido a posibles sedimentos que en ella se acumulen. En este análisis mucho tiene que ver la cercanía de estos tramos a cañadas, quebradas, arroyos, entre otros, para facilitar el drenaje.

\section{CONCLUSIONES}

La obtención de redes cuasi-abiertas en los métodos clásicos de optimización se debe a que no consideran los costos variables producto del consumo energético y cuando se 
tienen en cuenta, simplifican el modelo en la búsqueda de un óptimo global que no es funcional. La simplificación del modelo se efectúa para realzar considerablemente la convergencia del sistema no lineal de ecuaciones, donde no se tiene en cuenta el valor del diámetro como variable de decisión. Sin embargo, el incremento del valor de esta variable influye negativamente desde el punto de vista económico, pero a su vez, disminuye el valor de las pérdidas energéticas, como consecuencia al comportamiento real del modelo, que no es monótono creciente. Considerar como variable de decisión al diámetro de la tubería conlleva a la obtención de un modelo cóncavo y multimodal para cuya solución deberán emplearse técnicas heurísticas.

La optimización del proceso de diseño debe desarrollarse de modo sistémico para poder enfrentar el problema con toda su complejidad. En estos casos mucho tiene que ver el Análisis y la Síntesis de Sistemas de Ingeniería, lo cual permite insertar adecuadamente la tarea de estudio al sistema de mayor envergadura dentro del que se inscriben las redes de distribución de agua.

Para la optimización del diseño de redes de distribución de agua con topología mallada o mixta, se decide aplicar el método de Integración de Variables por ser una metaheurística evolutiva, al igual que las utilizadas tradicionalmente en las tareas de optimación del objeto de esta investigación.

Se considera incluir como indicador de eficiencia, las pérdidas de energía (resultado de la simulación hidráulica). De este modo se puede evaluar adecuadamente la eficiencia energética durante la actividad de diseño de las redes de distribución de agua, independientemente del sistema de suministro (inyección o regulación).

\section{REFERENCIAS BIBLIOGRÁFICAS}

Aguirre, A. et al. (1996): "Ingeniería Hidráulica aplicada a los sistemas de distribución de agua", Vol. I, eds. U.D. Mecánica de Fluidos - Universidad Poliectica de Valencia Aguas de Valencia S.A., Valencia, España.

Haestad (2009): "User's manual WaterCAD 8.0", Water Distribution Modeling \& Management Software. Haestad Methods, USA. http://www.bentley.com

ISSN: 1390-9320, Vol. 4, No. 3, junio 2017 
Hechavarría H, JR (2006). "Aplicación de tecnologías de avanzada en la rehabilitación de la red de abasto de la Ciudad de Camagüey". VIII Congreso de Hidráulica, Isla de la Juventud, Cuba. ISBN 978-959-247-036-1.

Hechavarría H, JR (2011): "Análisis y síntesis de redes de distribución de agua para la optimización del diseño multiobjetivo". X Congreso Internacional de Ingeniería Hidráulica. Grupo 11: Obras Hidráulicas: Economía, Diseño y Construcción. Editorial Obras, Holguín, Cuba. ISBN 978-959-247-082-8.

Hechavarría H, J. R.; Arzola R., J.; Cordovés G, A. y Lastre A., A (2007) "Diseño Óptimo de Redes Hidráulicas bajo Criterios Múltiples". Revista Ingeniería Mecánica, vol. 10, n. 2, pp. 15-22, CUJAE, Cuba. ISNN 1815-5944.

Hechavarría H, JR; Arzola R, J.; Cordovés G, A. y Lastre A, A. (2010) "Optimización del diseño de redes de distribución bajo criterios múltiples". Libro: Planificación, proyecto y operación de sistemas de abastecimiento de agua. Grupo Flulng - IMM - UPV. Valencia, España, pp. 37. ISBN 978-84-89487-31-4.

Montero L, R. (2016): “Optimización energética de la operación de los sistemas de climatización por agua helada en hoteles”. Revista Inteligencia Artificial 18(56), pp. 43-46, doi: 10.4114/ia.v18i56.1125. ISSN 1988-3064.

Montero L, R.; Legrá L, A. y Hechavarría H, JR (2016): “Optimización operacional de redes hidráulicas para climatización centralizada de hoteles”. Ingeniería Hidráulica y Ambiental, CUJAE, Cuba. riha, vol.37, n.2, pp.3-17. ISSN 1680-0338.

Rossman, L. (2001): “EPANET 2.0 Users Manual”. Water Supply and Water Resources Division, National Risk Management Research Laboratory, Office of Research and Development, U.S. Environmental Protection Agency, Cincinnati, OH 45268. 
Streeter, V., Benjamin, E. and Bedford, K. (2000): "Mecánica de Fluidos", Novena Edición. McGraw-Hill. Best Seller International, S.A., Santafé de Bogotá, Colombia. http://www.mhhe.com

Walski, T.M. (2001): "The wrong paradigm - Why water distribution optimization doesn't work", J. Water Resour. Plan. Manage, 127(4), 203-205

Licencia Creative Commons

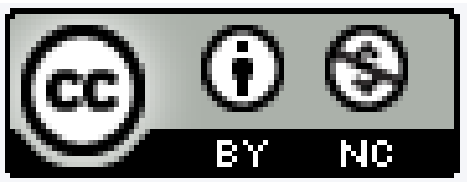

Revista Científica ECOCIENCIA está bajo una Licencia Creative Commons Atribución-NoComercial 4.0 Internacional. 